\title{
Some basic properties and fundamental relations for discrete Muckenhoupt and Gehring classes
}

\author{
S.H. Saker ${ }^{1,2}$, S.S. Rabie ${ }^{2}$, Jehad Alzabut ${ }^{3^{*}}$ (D, D. O'Regan ${ }^{4}$ and R.P. Agarwal ${ }^{5}$
}

"Correspondence:

jalzabut@psu.edu.sa

${ }^{3}$ Department of Mathematics and

General Sciences, Prince Sultan

University, 11586 Riyadh, Saudi

Arabia

Full list of author information is

available at the end of the article

\begin{abstract}
In this paper, we prove some basic properties of the discrete Muckenhoupt class $\mathcal{A}^{p}$ and the discrete Gehring class $\mathcal{G}^{q}$. These properties involve the self-improving properties and the fundamental transitions and inclusions relations between the two classes.
\end{abstract}

MSC: Primary 40D05; 40D25; secondary 42C10; 43A55; 46A35; 46B15

Keywords: Discrete Gehring's class; Discrete Muchenhoupt's class; Self-improving properties

properties

\section{Springer}

\section{Introduction}

The study of the discrete analogues in harmonic analysis became an active field of research in recent years. For example, the study of regularity and boundedness of discrete operators on $l^{p}$ analogues for $L^{p}$-regularity and higher summability of sequences has been considered by some authors, see for example $[3-7,24,25,28]$ and the references cited therein. Whereas some results from Euclidean harmonic analysis admit an obvious variant in the discrete setting, others do not. The main challenge in such studies is that there are no general methods to study these questions and the discrete operators may behave differently from their continuous counterparts as is exhibited by the discrete spherical maximal operator [19]. We confine ourselves in proving some basic properties of discrete Muckenhoupt and discrete Gehring classes. For properties and the structure of classical Muckenhoupt and Gehring classes (in integral forms), the relation between them and their applications in mathematical analysis, we refer the reader to the papers [1, 2, 8-18, 20-23, 27, 29] and the references cited therein. Throughout this paper, we assume that $1<p<\infty$ and II stands for a subset of $\mathbb{Z}_{+}$of the form $\mathbb{I}=\{1,2, \ldots, N\}$, where $N$ is a fixed positive integer and $n$ be a positive integer in $\mathbb{I}$. A discrete weight on $\mathbb{Z}_{+}=\{1,2, \ldots\}$ is a sequence $u=\{u(n)\}_{n=1}^{\infty}$ of positive real numbers. The discrete positive weight $u$ is said to belong to the discrete Muckenhoupt class $\mathcal{A}^{p}(A)$ on the interval $\mathbb{I}$, for $p>1$ and $A>1$ (independent of $p$ ), if, for

(c) The Author(s) 2020. This article is licensed under a Creative Commons Attribution 4.0 International License, which permits use, sharing, adaptation, distribution and reproduction in any medium or format, as long as you give appropriate credit to the original author(s) and the source, provide a link to the Creative Commons licence, and indicate if changes were made. The images or other third party material in this article are included in the article's Creative Commons licence, unless indicated otherwise in a credit line to the material. If material is not included in the article's Creative Commons licence and your intended use is not permitted by statutory regulation or exceeds the permitted use, you will need to obtain permission directly from the copyright holder. To view a copy of this licence, visit http://creativecommons.org/licenses/by/4.0/. 
every $n \in \mathbb{I}$,

$$
\frac{1}{n} \sum_{k=1}^{n} u(k) \leq A\left(\frac{1}{n} \sum_{k=1}^{n} u^{\frac{1}{1-p}}(k)\right)^{1-p} .
$$

For a given exponent $p>1$, we define the $\mathcal{A}^{p}$-norm of the discrete weight $u$ by the following quantity:

$$
[u]_{\mathcal{A}^{p}}:=\sup _{n \in \mathbb{I}}\left(\frac{1}{n} \sum_{k=1}^{n} u(k)\right)\left(\frac{1}{n} \sum_{k=1}^{n} u^{\frac{-1}{p-1}}(k)\right)^{p-1},
$$

where the supremum is taken over all $n \in \mathbb{I}$. The discrete weight $u$ is said to belong to the discrete Muckenhoupt class $\mathcal{A}^{1}(A)$ if, for every $n \in \mathbb{I}$,

$$
\frac{1}{n} \sum_{k=1}^{n} u(k) \leq A \inf _{1 \leq k \leq n} u(k)
$$

for $A>1$, holds and we define the $\mathcal{A}^{1}$-norm by the following quantity:

$$
[u]_{\mathcal{A}^{1}}=\sup _{n \in \mathbb{I}} \frac{1}{n}\left(\frac{1}{\inf _{1 \leq k \leq n} u(k)} \sum_{k=1}^{n} u(k)\right) .
$$

The discrete weight $u$ is said to belong to the discrete Muckenhoupt class $\mathcal{A}^{\infty}(A)$ if, for every $n \in \mathbb{I}$,

$$
\left(\frac{1}{n} \sum_{k=1}^{n} u(k)\right)\left(\exp \frac{1}{n} \sum_{k=1}^{n} \log \frac{1}{u(k)}\right) \leq A,
$$

for $A>1$, and we define the $\mathcal{A}^{\infty}$-norm by the following quantity:

$$
[u]_{\mathcal{A}^{\infty}}:=\sup _{n \in \mathbb{I}}\left(\frac{1}{n} \sum_{k=1}^{n} u(k)\right)\left(\exp \frac{1}{n} \sum_{k=1}^{n} \log \frac{1}{u(k)}\right),
$$

where the supremum is taken over all $n \in \mathbb{I}$. The discrete positive weight $u$ is said to belong to the discrete Gehring class $\mathcal{G}^{q}(G)$ on the interval $\mathbb{I} \subset \mathbb{Z}^{+}$, for $q>1$ and $G>1$ (independent of $q$ ), if, for every $n \in \mathbb{I}$,

$$
\left(\frac{1}{n} \sum_{k=1}^{n} u^{q}(k)\right)^{1 / q} \leq G \frac{1}{n} \sum_{k=1}^{n} u(k) .
$$

For a given exponent $q>1$, we define the $\mathcal{G}^{q}$-norm by the following quantity:

$$
[u]_{\mathcal{G}^{q}}:=\sup _{n \in \mathbb{I}}\left[\left(\frac{1}{n} \sum_{k=1}^{n} u(k)\right)^{-1}\left(\frac{1}{n} \sum_{k=1}^{n} u^{q}(k)\right)^{\frac{1}{q}}\right]^{\frac{q}{q-1}}
$$


where the supremum is taken over all $n \in \mathbb{I}$. The discrete weight $u$ is said to belong to the discrete Gehring class $\mathcal{G}^{\infty}(G)$ if for every $n \in \mathbb{I}$

$$
\sup _{1 \leq k \leq n} u(k) \leq G \frac{1}{n} \sum_{k=1}^{n} u(k)
$$

for $G>0$. The discrete weight is said to belong to the discrete Gehring class $\mathcal{G}^{1}(G)$ if, for every $n \in \mathbb{I}$,

$$
\exp \left(\frac{1}{n} \sum_{k=1}^{n} \frac{u(k)}{\frac{1}{n} \sum_{k=1}^{n} u(k)} \log \frac{u(k)}{\frac{1}{n} \sum_{k=1}^{n} u(k)}\right) \leq G,
$$

where $G>1$ and we define the $\mathcal{G}^{1}$-norm by the following quantity:

$$
[u]_{\mathcal{G}^{1}}:=\sup _{n \in \mathbb{I}}\left[\exp \left(\frac{1}{n} \sum_{k=1}^{n} \frac{u(k)}{\frac{1}{n} \sum_{k=1}^{n} u(k)} \log \frac{u(k)}{\frac{1}{n} \sum_{k=1}^{n} u(k)}\right)\right],
$$

where the supremum is taken over all $n \in \mathbb{I}$. The objective of this paper is classified as follows:

(1) Prove some basic properties of the discrete Muckenhoupt class $\mathcal{A}^{p}$.

(2) Prove some basic properties of the discrete Gehring class $\mathcal{G}^{q}$.

(3) Prove some fundamental relations between $\mathcal{A}^{p}$ and $\mathcal{G}^{q}$.

(4) Prove some fundamental relations between $\mathcal{A}^{\infty}$ and $\mathcal{G}^{q}$ and their norms.

The paper is organized as follows: In the next section, we state and prove some basic lemmas that are needed in the proofs of the main results. In Sect. 3, we present properties of the Muckenhoupt class which include the self-improving property. In Sect. 4, we prove the basic properties of the Gehring class which include also the self-improving property. In Sect. 5, we prove the transition and inclusion relations between the two classes which give embedding relations between $\mathcal{A}^{p}$ and $\mathcal{G}^{q}$ and also relations between $\mathcal{A}^{\infty}$ and $\mathcal{G}^{q}$ and their norms.

\section{Basic lemmas}

In what follows, all sequences in the statements of the theorems are assumed to be positive sequences defined on $\mathbb{I} \subseteq \mathbb{Z}_{+}$and use the conventions $0 \cdot \infty=0$ and $0 / 0=0$ and $\sum_{k=m}^{b} y(k)=0$, whenever $m>b$ and

$$
\Delta\left(\sum_{s=a}^{k-1} y(s)\right)=y(k), \quad \sum_{s=a}^{k-1} \Delta y(s)=y(k)-y(a) .
$$

The classical Hölder inequality is given by

$$
\sum_{k=1}^{n}|u v| \leq\left[\sum_{k=1}^{n}|u|^{p}\right]^{1 / p}\left[\sum_{k=1}^{n}|v|^{q}\right]^{1 / q},
$$

where $1 / p+1 / q=1$, and $p, q>1$. This inequality is reversed for $0<p<1$ and if $p<0$ or $q<0$. For instance the inequality

$$
\sum_{k=1}^{n}|u v| \geq\left[\sum_{k=1}^{n}|u|^{1 / p}\right]^{p}\left[\sum_{k=1}^{n}|v|^{-1 /(p-1)}\right]^{-(p-1)},
$$


holds if $0<p<1$ or $p>1, q=-1 /(p-1)<0$. If $p=1$ and $q=\infty$, the Hölder inequality is given by

$$
\sum_{k=1}^{n}|u v| \leq\left(\sum_{k=1}^{n}|u|\right)\left(\sup _{n}|v|\right),
$$

if $\sum_{k=1}^{n}|u(k)|<\infty$, and $\sup _{n}|v(n)|<\infty$. Throughout we assume that $u: \mathbb{I} \rightarrow \mathbb{R}^{+}$is positive sequence and define

$$
\mathcal{M}_{q} u(n):=\left(\frac{1}{n} \sum_{k=1}^{n} u^{q}(k)\right)^{1 / q},
$$

for any real number $q$ and any $n \in \mathbb{I}$. Note that, for $q=0$, the operator $\mathcal{M}_{q}$ takes the form

$$
\mathcal{M}_{0} u=\exp \left(\frac{1}{n} \sum_{k=1}^{n} \log u(k)\right) .
$$

In the following lemma, we state the basic property of the operator $\mathcal{M}_{q} u$ which is proved directly by applying Jensen's inequality.

Lemma 1 Let $u$ be a positive weight and $p$ and $q$ be real numbers. If $p \leq q$, then $\mathcal{M}_{p} u \leq$ $\mathcal{M}_{q} u$.

We recall that the discrete positive weight $u$ is said to belong to the discrete Gehring class $\mathcal{G}^{q}(G)$ on the interval $\mathbb{I} \subset \mathbb{Z}^{+}$, for $q>1$ and $G>1$, if, for every $n \in \mathbb{I}$,

$$
\left(\frac{1}{n} \sum_{k=1}^{n} u^{q}\right)^{1 / q} \leq G\left(\frac{1}{n} \sum_{k=1}^{n} u\right) .
$$

It is clear that the Hölder inequality (in terms of $\mathcal{M}_{q} u$ ) reads

$$
\mathcal{M}_{q} u \geq \mathcal{M}_{1} u, \quad \text { for } q \geq 1 .
$$

The reverse of (7) is given by (6), which in terms of $\mathcal{M}$ reads

$$
\mathcal{M}_{q} u \leq G \mathcal{M}_{1} u
$$

for some constant $G>1$. A generalization of inequality (8) for $1<p \leq q$, which we call the generalized reverse Hölder inequality, is given in terms of $\mathcal{M}$ by

$$
\mathcal{M}_{q} u \leq G \mathcal{M}_{p} u
$$

for some constant $G>1$. In [26], the authors proved the following transition properties which gives a transition relation between the class $\mathcal{A}^{p}$ and the class $\mathcal{G}^{q}$.

Theorem 2 Let $u$ be a positive weight and $p$ and $q$ be real numbers. Then $u \in \mathcal{A}^{p}$ for some $p$ if and only if $u \in \mathcal{G}^{q}$ for some $q$. 
Remark 1 The equivalence in this theorem gives the transition property between the discrete Muckenhoupt and Gehring classes. The main question which is interesting is what the relation is between $p$ and $q$ for which the inclusions $\mathcal{A}^{p} \subset \mathcal{G}^{q}$ and $\mathcal{G}^{q} \subset \mathcal{A}^{p}$ hold, and this remains an open problem.

\section{Some basic properties of Muckenhoupt weights}

In this section, we prove some basic properties of Muckenhoupt weights. The first lemma proves the inclusion of the class $\mathcal{A}^{p}$ in the class $\mathcal{A}^{\infty}$ for all $p \geq 1$. That is, if $u \in \mathcal{A}^{p}$ for $p \geq 1$, then $u \in \mathcal{A}^{\infty}$.

Lemma 3 Let $u$ be a positive weight and $p$ be a nonnegative real number. If $u \in \mathcal{A}^{p}(A)$, then the inequality

$$
\mathcal{M}_{1} u \leq A \exp \left(\mathcal{M}_{1} \log u\right)
$$

holds.

Proof Since $u \in \mathcal{A}^{p}(A)$, then, for all $n \in \mathbb{I}$ and $A>1$, we have

$$
\frac{1}{n} \sum_{k=1}^{n} u(k) \leq A\left(\frac{1}{n} \sum_{k=1}^{n} u^{\frac{1}{1-p}}(k)\right)^{1-p} .
$$

By taking the limit as $p$ tends to $\infty$, then the right hand side, after using the properties of limits and L'Hôpital's rule, becomes

$$
\frac{1}{n} \sum_{k=1}^{n} u \leq A \lim _{p \rightarrow \infty}\left(\frac{1}{n} \sum_{k=1}^{n} u^{\frac{1}{1-p}}(k)\right)^{1-p}=A \exp \left(\frac{1}{n} \sum_{k=1}^{n} \log u(k)\right),
$$

which is the desired inequality (10). The proof is complete.

Theorem 4 Let $u$ be a positive weight and $p$ be a nonnegative real number, and $p^{\prime}=p /(p-$ 1) be the conjugate of $p$. Then $u \in \mathcal{A}^{p}$ if and only if $u^{1-p^{\prime}} \in \mathcal{A}^{p^{\prime}}$, with

$$
\left[u^{1-p^{\prime}}\right]_{\mathcal{A}^{p^{\prime}}}=[u]_{\mathcal{A}^{p}}^{p^{\prime}-1}
$$

Proof From the definition of the class $\mathcal{A}^{p}$, and since $1-p^{\prime}=1 /(1-p)<0$, we have for $A>1$ and all $n \in \mathbb{I}$

$$
\begin{aligned}
u \in \mathcal{A}^{p} & \Leftrightarrow \frac{1}{n} \sum_{k=1}^{n} u(k) \leq A\left(\frac{1}{n} \sum_{k=1}^{n} u^{\frac{1}{1-p}}(k)\right)^{1-p} \\
& \Leftrightarrow\left(\frac{1}{n} \sum_{k=1}^{n} u(k)\right)^{\frac{1}{1-p}} \geq A^{\frac{1}{1-p}} \frac{1}{n} \sum_{k=1}^{n} u^{\frac{1}{1-p}}(k) \\
& \Leftrightarrow \frac{1}{n} \sum_{k=1}^{n} u^{1-p^{\prime}}(k) \leq A^{p^{\prime}-1}\left(\frac{1}{n} \sum_{k=1}^{n}\left(u^{1-p^{\prime}}(k)\right)^{\frac{1}{1-p^{\prime}}}\right)^{1-p^{\prime}} \\
& \Leftrightarrow u^{1-p^{\prime}} \in \mathcal{A}^{p^{\prime}} .
\end{aligned}
$$


Furthermore, since $\frac{1}{1-p}=1-p^{\prime}$ and $\frac{1}{1-p^{\prime}}=1-p$, we have

$$
\begin{aligned}
\sup _{n \in \mathbb{I}}\left(\frac{1}{n} \sum_{k=1}^{n} u^{1-p^{\prime}}(k)\right)\left(\frac{1}{n} \sum_{k=1}^{n}\left(u^{1-p^{\prime}}(k)\right)^{\frac{1}{1-p^{\prime}}}\right)^{-\left(1-p^{\prime}\right)} \\
=\sup _{n \in \mathbb{I}}\left(\frac{1}{n} \sum_{k=1}^{n} u^{1 /(1-p)}(k)\right)^{\left(p^{\prime}-1\right)(p-1)}\left(\frac{1}{n} \sum_{k=1}^{n} u(k)\right)^{-\left(1-p^{\prime}\right)} \\
=\sup _{n \in \mathbb{I}}\left[\left(\frac{1}{n} \sum_{k=1}^{n} u^{1 /(1-p)}(k)\right)^{p-1}\left(\frac{1}{n} \sum_{k=1}^{n} u(k)\right)\right]^{p^{\prime}-1} .
\end{aligned}
$$

This is equivalent to

$$
\left[u^{1-p^{\prime}}\right]_{\mathcal{A}^{p^{\prime}}}=[u]_{\mathcal{A}^{p}}^{p^{\prime}-1}
$$

which is the desired result. The proof is complete.

In the next theorem, we prove some basic inclusion properties of Muckenhoupt classes.

Theorem 5 Let $u$ be a positive weight and $p, q$ be nonnegative real numbers. Then the following inclusion relations hold:

(1) $\mathcal{A}^{1} \subset \mathcal{A}^{p} \subset \mathcal{A}^{\infty}$, for all $1<p<\infty$,

(2) $\mathcal{A}^{p} \subset \mathcal{A}^{q}$ for all $1<p \leq q$,

(3) $\mathcal{A}^{\infty}=\bigcup_{1<p} \mathcal{A}^{p}$ with

$$
[u]_{\mathcal{A}^{\infty}}=\lim _{p \rightarrow \infty}[u]_{\mathcal{A}^{p}}
$$

and $\mathcal{A}^{1} \subset \bigcap_{p>1} \mathcal{A}^{p}$.

Proof (1) Let $u \in \mathcal{A}^{1}$, then there exists $A>1$ such that, for all $n \in \mathbb{I}$, we have

$$
\frac{1}{n} \sum_{k=1}^{n} u(k) \leq A \inf _{1 \leq k \leq n} u(k)
$$

or equivalently

$$
\frac{1}{n} \sum_{k=1}^{n} u(k) \leq A u(k)
$$

for all $1 \leq k \leq n$. For $p>1$, by using (11) we have, for all $n \in \mathbb{I}$,

$$
\begin{aligned}
\left(\frac{1}{n} \sum_{k=1}^{n} u(k)\right)\left(\frac{1}{n} \sum_{k=1}^{n} u^{\frac{1}{1-p}}(k)\right)^{p-1} & \leq\left(\frac{1}{n} \sum_{k=1}^{n} u(k)\right)\left(\frac{1}{n} \sum_{k=1}^{n}\left(A^{-1} \frac{1}{n} \sum_{k=1}^{n} u(k)\right)^{\frac{1}{1-p}}\right)^{p-1} \\
& =\left(\frac{1}{n} \sum_{k=1}^{n} u(k)\right)\left(A^{-1} \frac{1}{n} \sum_{k=1}^{n} u(k)\right)^{-1}=A .
\end{aligned}
$$


Hence $u \in \mathcal{A}^{p}$, which implies that $\mathcal{A}^{1} \subset \mathcal{A}^{p}$. Now, assume that $u \in \mathcal{A}^{p}$, then Lemma 3 implies that $u \in \mathcal{A}^{\infty}$. That is $\mathcal{A}^{p} \subset \mathcal{A}^{\infty}$.

(2) Assume that $u \in \mathcal{A}^{p}$, then there exists $A>1$ such that for all $n \in \mathbb{I}$

$$
\frac{1}{n} \sum_{k=1}^{n} u(k)\left(\frac{1}{n} \sum_{k=1}^{n} u^{\frac{1}{1-p}}(k)\right)^{p-1} \leq A,
$$

holds. Now, since $1<p \leq q$, we see that $\frac{1}{p-1} \geq \frac{1}{q-1}$, and then, by using Lemma 1 , we have

$$
\mathcal{M}_{\frac{1}{p-1}} u^{-1} \geq \mathcal{M}_{\frac{1}{q-1}} u^{-1}
$$

Then, for all $n \in \mathbb{I}$, we obtain

$$
\begin{aligned}
A & \geq \frac{1}{n} \sum_{k=1}^{n} u(k)\left(\frac{1}{n} \sum_{k=1}^{n} u^{\frac{1}{1-p}}(k)\right)^{p-1} \\
& \geq \frac{1}{n} \sum_{k=1}^{n} u(k)\left(\frac{1}{n} \sum_{k=1}^{n} u^{\frac{1}{1-q}}(k)\right)^{q-1},
\end{aligned}
$$

which implies that $u \in \mathcal{A}^{q}$.

(3) By applying Property (1), for all $1<p<\infty, \mathcal{A}^{p} \subset \mathcal{A}^{\infty}$. Then,

$$
\bigcup_{1 \leq p<\infty} \mathcal{A}^{p} \subseteq \mathcal{A}^{\infty}
$$

Conversely, we shall prove the containment by contradiction. That is, we assume that $u \in$ $\mathcal{A}^{\infty}$ and assume, on the contrary, that, for all $1 \leq p<\infty, u \notin \mathcal{A}^{p}$. Then, for all $1 \leq p<\infty$, we see that

$$
\sup _{n \in \mathbb{I}}\left(\frac{1}{n} \sum_{k=1}^{n} u(k)\right)\left(\frac{1}{n} \sum_{k=1}^{n} u^{\frac{1}{1-p}}(k)\right)^{p-1}=\infty
$$

which, by taking the limit as $p$ tends to $\infty$ implies that

$$
\sup _{n \in \mathbb{I}}\left(\frac{1}{n} \sum_{k=1}^{n} u\right)\left(\exp \frac{1}{n} \sum_{k=1}^{n} \log \frac{1}{u(k)}\right)=\infty .
$$

This contradicts the assumption that $u \in \mathcal{A}^{\infty}$, then $u \in \mathcal{A}^{\infty}$ implies that, for some $1 \leq p<$ $\infty, u \in \mathcal{A}^{p}$ and hence

$$
u \in \bigcup_{1 \leq p<\infty} \mathcal{A}^{p}
$$

Thus

$$
\mathcal{A}^{\infty} \subseteq \bigcup_{1 \leq p<\infty} \mathcal{A}^{p}
$$


From (12) and (13), we obtain $\mathcal{A}^{\infty}=\bigcup_{1 \leq p<\infty} \mathcal{A}^{p}$. Moreover, by applying L'Hôpital's rule and some limit rules, we obtain

$$
\begin{aligned}
\lim _{p \rightarrow \infty}[u]_{\mathcal{A}^{p}} & =\lim _{p \rightarrow \infty} \sup _{n \in \mathbb{I}}\left(\frac{1}{n} \sum_{k=1}^{n} u(k)\right)\left(\frac{1}{n} \sum_{k=1}^{n} u^{\frac{1}{1-p}}(k)\right)^{p-1} \\
& =\sup _{n \in \mathbb{I}} \lim _{p \rightarrow \infty}\left(\frac{1}{n} \sum_{k=1}^{n} u(k)\right)\left(\frac{1}{n} \sum_{k=1}^{n} u^{\frac{1}{1-p}}(k)\right)^{p-1} \\
& =\sup _{n \in \mathbb{I}}\left(\frac{1}{n} \sum_{k=1}^{n} u(k)\right)\left(\exp \frac{1}{n} \sum_{k=1}^{n} \log \frac{1}{u(k)}\right) \\
& =[u]_{\mathcal{A}}^{\infty},
\end{aligned}
$$

which is the desired result. Now, assume that $u \in \mathcal{A}^{1}$. By Property (1), for any $p>1, \mathcal{A}^{1} \subset$ $\mathcal{A}^{p}$, then

$$
\mathcal{A}^{1} \subset \bigcap_{p>1} \mathcal{A}^{p} .
$$

Equality does not hold and to prove it is sufficient to provide an example of a weight $u$ satisfies $u \in \bigcap_{p>1} \mathcal{A}^{p} \backslash \mathcal{A}^{1}$. For example: for all $p>1$, we have $u(n)=n^{\alpha} \in \mathcal{A}^{p}$ for $\alpha>1$ and $u(n) \notin \mathcal{A}^{1}$. The proof is complete.

Remark 2 In Theorem 5, we were able to prove the containment $\mathcal{A}^{1} \subset \bigcap_{p>1} \mathcal{A}^{p}$. In the following, we present some weights, which does not only satisfy the containment $\mathcal{A}^{1} \subset$ $\bigcap_{p>1} \mathcal{A}^{p}$ but also satisfy the equalities:

(i) $u(n)=1 \in \mathcal{A}^{1}$ and hence $u(n) \in \mathcal{A}^{p}$,

(ii) $u(n)=n^{\alpha} \in \mathcal{A}^{1}$ for $\alpha \leq 0$ and hence $u(n) \in \mathcal{A}^{p}$,

(iii) $u(n)=\frac{1}{\log (n+1)} \in \mathcal{A}^{1}$ and hence $u(n) \in \mathcal{A}^{p}$.

In the following theorem, we discuss the power rule for weights in the Muckenhoupt class. That is, we discuss the cases for $\alpha$ which satisfies the necessity of the statement: $u \in \mathcal{A}^{p}$ implies that $u^{\alpha} \in \mathcal{A}^{p}$.

Theorem 6 Let $u$ be a positive weight, $p$ be a nonnegative real number. Then

(1) if $u \in \mathcal{A}^{p}$ then $u^{\alpha} \in \mathcal{A}^{p}$, for $0 \leq \alpha \leq 1$, with $\left[u^{\alpha}\right]_{\mathcal{A}^{p}} \leq[u]_{\mathcal{A}^{p}}^{\alpha}$,

(2) if $u \in \mathcal{A}^{p}$, then $u^{\alpha} \in \mathcal{A}^{p}$ for some $\alpha>1$.

Proof (1) For $0 \leq \alpha \leq 1$, and $u \in \mathcal{A}^{p}$, we have $\frac{1}{p-1} \geq \frac{\alpha}{p-1}>0$, and hence by applying Lemma 1 , we have for all $n \in \mathbb{I}$

$$
\begin{aligned}
& \left(\frac{1}{n} \sum_{k=1}^{n} u^{\alpha}(k)\right)\left(\frac{1}{n} \sum_{k=1}^{n}\left(u^{\alpha}(k)\right)^{\frac{1}{1-p}}\right)^{p-1} \\
& \leq\left(\frac{1}{n} \sum_{k=1}^{n} u(k)\right)^{\alpha}\left(\frac{1}{n} \sum_{k=1}^{n} u^{\frac{\alpha}{1-p}}(k)\right)^{p-1}
\end{aligned}
$$




$$
\begin{aligned}
& \leq\left(\frac{1}{n} \sum_{k=1}^{n} u(k)\right)^{\alpha}\left(\frac{1}{n} \sum_{k=1}^{n} u^{\frac{1}{1-p}}(k)\right)^{\alpha(p-1)} \\
& =\left[\left(\frac{1}{n} \sum_{k=1}^{n} u(k)\right)\left(\frac{1}{n} \sum_{k=1}^{n} u^{\frac{1}{1-p}}(k)\right)^{(p-1)}\right]^{\alpha} \leq A^{\alpha},
\end{aligned}
$$

that is, $u^{\alpha} \in \mathcal{A}^{p}$, with $\left[u^{\alpha}\right]_{\mathcal{A}^{p}} \leq[u]_{\mathcal{A}^{p}}^{\alpha}$, which is the desired result.

(2) Since $u \in \mathcal{A}^{p}(A)$,

$$
\left(\frac{1}{n} \sum_{k=1}^{n} u(k)\right) \leq A\left(\frac{1}{n} \sum_{k=1}^{n} u^{1 /(1-p)}(k)\right)^{1-p}, \quad A \geq 1 .
$$

Theorem 2 implies that $u \in \mathcal{G}^{q}(G)$. That is,

$$
\left(\frac{1}{n} \sum_{k=1}^{n} u^{q}(k)\right)^{1 / q} \leq G\left(\frac{1}{n} \sum_{k=1}^{n} u(k)\right)
$$

Also, Theorem 4 implies that $u^{1 /(1-p)} \in \mathcal{A}^{p^{\prime}}$, again Theorem 2 implies that $u^{1 /(1-p)} \in$ $\mathcal{G}^{q_{1}}\left(G_{1}\right)$. That is,

$$
\left(\frac{1}{n} \sum_{k=1}^{n} u^{q_{1} /(1-p)}(k)\right)^{1 / q_{1}} \leq G_{1}\left(\frac{1}{n} \sum_{k=1}^{n} u^{1 /(1-p)}(k)\right) .
$$

Now, by applying (14), (15), and (16), we have

$$
\begin{aligned}
\frac{1}{n} \sum_{k=1}^{n} u^{q}(k) & \leq G^{q}\left(\frac{1}{n} \sum_{k=1}^{n} u(k)\right)^{q} \\
& \leq G^{q}\left[A\left(\frac{1}{n} \sum_{k=1}^{n} u^{1 /(1-p)}(k)\right)^{1-p}\right]^{q} \\
& =G^{q} A^{q}\left(\frac{1}{n} \sum_{k=1}^{n} u^{1 /(1-p)}(k)\right)^{q(1-p)} \\
& \leq G^{q} A^{q}\left[G_{1}^{-1}\left(\frac{1}{n} \sum_{k=1}^{n} u^{q_{1} /(1-p)}(k)\right)^{1 / q_{1}}\right]^{q(1-p)} \\
& =G^{q} A^{q} G_{1}^{q(p-1)}\left(\frac{1}{n} \sum_{k=1}^{n} u^{q_{1} /(1-p)}(k)\right)^{q(1-p) / q_{1}} .
\end{aligned}
$$

If $q<q_{1}$, then, by applying Lemma 1 , it satisfies

$$
\left(\frac{1}{n} \sum_{k=1}^{n} u^{q_{1} /(1-p)}(k)\right)^{q(1-p) / q_{1}} \leq\left(\frac{1}{n} \sum_{k=1}^{n} u^{q /(1-p)}(k)\right)^{(1-p)},
$$


which implies that

$$
\frac{1}{n} \sum_{k=1}^{n} u^{q}(k) \leq G^{q} A^{q} G_{1}^{q(p-1)}\left(\frac{1}{n} \sum_{k=1}^{n} u^{q /(1-p)}(k)\right)^{(1-p)},
$$

or equivalently, $u^{q} \in \mathcal{A}^{p}$. If $q>q_{1}$, then by using Hölder's inequality we see that the condition $u \in \mathcal{G}^{q}$ implies that

$$
\left(\frac{1}{n} \sum_{k=1}^{n} u^{q_{1}}(k)\right)^{1 / q_{1}} \leq G_{2}\left(\frac{1}{n} \sum_{k=1}^{n} u(k)\right) .
$$

By using (14), (16) and (17), we have

$$
\begin{aligned}
\frac{1}{n} \sum_{k=1}^{n} u^{q_{1}}(k) & \leq G_{2}^{q_{1}}\left(\frac{1}{n} \sum_{k=1}^{n} u(k)\right)^{q_{1}} \\
& \leq G_{2}^{q_{1}}\left[A\left(\frac{1}{n} \sum_{k=1}^{n} u^{1 /(1-p)}(k)\right)^{1-p}\right]^{q_{1}} \\
& =G_{2}^{q_{1}} A^{q_{1}}\left(\frac{1}{n} \sum_{k=1}^{n} u^{1 /(1-p)}(k)\right)^{(1-p) q_{1}} \\
& \leq G_{2}^{q_{1}} A^{q_{1}} G_{1}^{p-1}\left(\frac{1}{n} \sum_{k=1}^{n} u^{q_{1} /(1-p)}(k)\right)^{(1-p)}
\end{aligned}
$$

that is, $u^{q_{1}} \in \mathcal{A}^{p}$. This completes our proof.

Theorem 7 If $u_{1}, u_{2} \in \mathcal{A}^{p}$, then $u_{1}^{\alpha} u_{2}^{1-\alpha} \in \mathcal{A}^{p}, 0 \leq \alpha \leq 1$, with a constant

$$
\left[u_{1}^{\alpha} u_{2}^{1-\alpha}\right]_{\mathcal{A}^{p}} \leq\left[u_{1}\right]_{\mathcal{A}^{p}}^{\alpha}\left[u_{2}\right]_{\mathcal{A}^{p}}^{1-\alpha} .
$$

Proof Assume that $u_{1}, u_{2} \in \mathcal{A}^{p}$, then

$$
\frac{1}{n} \sum_{k=1}^{n} u_{1}(k)\left(\frac{1}{n} \sum_{k=1}^{n} u_{1}^{\frac{1}{1-p}}(k)\right)^{p-1} \leq A_{1}
$$

and

$$
\frac{1}{n} \sum_{k=1}^{n} u_{2}(k)\left(\frac{1}{n} \sum_{k=1}^{n} u_{2}^{\frac{1}{1-p}}(k)\right)^{p-1} \leq A_{2}
$$

hold for all $n \in \mathbb{I}$, where $A_{1}, A_{2}>1$. By applying Hölder's inequality (note that $0 \leq \alpha \leq 1$ ) with $1 / \alpha$ and $1 /(1-\alpha)$ and using (18) and (19), we obtain

$$
\begin{aligned}
& \frac{1}{n} \sum_{k=1}^{n} u_{1}^{\alpha}(k) u_{2}^{1-\alpha}(k) \\
& \quad \leq\left(\frac{1}{n} \sum_{k=1}^{n} u_{1}(k)\right)^{\alpha}\left(\frac{1}{n} \sum_{k=1}^{n} u_{2}(k)\right)^{1-\alpha}
\end{aligned}
$$




$$
\begin{aligned}
& \leq\left(A_{1}\left(\frac{1}{n} \sum_{k=1}^{n} u_{1}^{1-p}(k)\right)^{1-p}\right)^{\alpha}\left(A_{2}\left(\frac{1}{n} \sum_{k=1}^{n} u_{2}^{\frac{1}{1-p}}(k)\right)^{1-p}\right)^{1-\alpha} \\
& =A_{1}^{\alpha} A_{2}^{1-\alpha}\left[\left(\frac{1}{n} \sum_{k=1}^{n} u_{1}^{\frac{1}{1-p}}(k)\right)^{\alpha}\left(\frac{1}{n} \sum_{k=1}^{n} u_{2}^{\frac{1}{1-p}}(k)\right)^{1-\alpha}\right]^{1-p} .
\end{aligned}
$$

Again, by applying Hölder's inequality for $1 / \alpha$ and $1 /(1-\alpha)$ on the term

$$
\frac{1}{n} \sum_{k=1}^{n} u_{1}^{\frac{\alpha}{1-p}}(k) u_{2}^{\frac{1-\alpha}{1-p}}(k)
$$

we obtain

$$
\frac{1}{n} \sum_{k=1}^{n} u_{1}^{\frac{\alpha}{1-p}}(k) u_{2}^{\frac{1-\alpha}{1-p}}(k) \leq\left(\frac{1}{n} \sum_{k=1}^{n} u_{1}^{\frac{1}{1-p}}(k)\right)^{\alpha}\left(\frac{1}{n} \sum_{k=1}^{n} u_{2}^{\frac{1}{1-p}}(k)\right)^{1-\alpha} .
$$

By substituting (21) into (20) and since $1-p<0$, we have

$$
\begin{aligned}
\frac{1}{n} \sum_{k=1}^{n} u_{1}^{\alpha}(k) u_{2}^{1-\alpha}(k) & \leq A_{1}^{\alpha} A_{2}^{1-\alpha}\left[\frac{1}{n} \sum_{k=1}^{n} u_{1}^{\frac{\alpha}{1-p}}(k) u_{2}^{\frac{1-\alpha}{1-p}}(k)\right]^{1-p} \\
& =A_{1}^{\alpha} A_{2}^{1-\alpha}\left[\frac{1}{n} \sum_{k=1}^{n}\left(u_{1}^{\alpha}(k) u_{2}^{1-\alpha}(k)\right)^{\frac{1}{1-p}}\right]^{1-p} .
\end{aligned}
$$

Hence $u_{1}^{\alpha} u_{2}^{1-\alpha} \in \mathcal{A}^{p}$, for $0 \leq \alpha \leq 1$, with

$$
\left[u_{1}^{\alpha} u_{2}^{1-\alpha}\right]_{\mathcal{A}^{p}} \leq\left[u_{1}\right]_{\mathcal{A}^{p}}^{\alpha}\left[u_{2}\right]_{\mathcal{A}^{p}}^{1-\alpha}
$$

The proof is complete.

In the next theorem, we discuss the relation between an $\mathcal{A}^{p}$-weight and the product of two sequences in the $\mathcal{A}^{1}$-class of weights.

Theorem $8 u \in \mathcal{A}^{p}$ if and only if there exist $u_{1}, u_{2} \in \mathcal{A}^{1}$ such that $u=u_{1} u_{2}^{1-p}, 1<p<\infty$.

Proof First, we prove that if $u \in \mathcal{A}^{p}$, then $u=u_{1} u_{2}^{1-p}$ such that $u_{1}, u_{2} \in \mathcal{A}^{1}$, or equivalently we prove that $\mathcal{A}^{p} \subset \mathcal{A}^{1}\left(\mathcal{A}^{1}\right)^{1-p}$. Assume that $u \notin \mathcal{A}^{1}\left(\mathcal{A}^{1}\right)^{1-p}$, then for all $u_{1}$ and $u_{2}$ satisfying $u=u_{1} u_{2}^{1-p}$, then $u_{1} \notin \mathcal{A}^{1}$ and $u_{2} \notin \mathcal{A}^{1}$. Now, since $\inf _{k} u(k) \leq u(k)$ for all $k$. Then

$$
\begin{aligned}
& \frac{1}{n} \sum_{k=1}^{n} u(k)\left(\frac{1}{n} \sum_{k=1}^{n} u^{1-p}(k)\right)^{p-1} \\
& \quad=\left(\frac{1}{n} \sum_{k=1}^{n} u_{1}(k) u_{2}^{1-p}(k)\right)\left(\frac{1}{n} \sum_{k=1}^{n} u_{1}^{\frac{1}{1-p}}(k) u_{2}(k)\right)^{p-1} \\
& \quad \leq\left(\frac{1}{n} \sum_{k=1}^{n} u_{1}(k)\right)\left(\inf _{k} u_{2}(k)\right)^{1-p}
\end{aligned}
$$




$$
\begin{aligned}
& \times\left(\frac{1}{n} \sum_{k=1}^{n} u_{1}^{1-p}(k) u_{2}(k)\right)^{p-1}\left(\inf _{k} u_{1}(k)\right)^{-1} \\
= & \left(\frac{1}{\inf _{k} u_{1}(k)} \frac{1}{n} \sum_{k=1}^{n} u_{1}(k)\right)\left(\frac{1}{\inf _{k} u_{2}(k)} \frac{1}{n} \sum_{k=1}^{n} u_{2}(k)\right)^{p-1} .
\end{aligned}
$$

By the assumption that $u_{1} \notin \mathcal{A}^{1}$ and $u_{2} \notin \mathcal{A}^{1}$, then

$$
\begin{aligned}
& \sup _{n} \frac{1}{n} \sum_{k=1}^{n} u(k)\left(\frac{1}{n} \sum_{k=1}^{n} u^{\frac{1}{1-p}}(k)\right)^{p-1} \\
& \quad \leq \sup _{n}\left(\frac{1}{\inf _{k} u_{1}(k)} \frac{1}{n} \sum_{k=1}^{n} u_{1}(k)\right)\left(\frac{1}{\inf _{k} u_{2}(k)} \frac{1}{n} \sum_{k=1}^{n} u_{2}(k)\right)^{p-1} \\
& \quad=\infty .
\end{aligned}
$$

That is $u \notin \mathcal{A}^{p}$. That is $\mathcal{A}^{p} \subset \mathcal{A}^{1}\left(\mathcal{A}^{1}\right)^{1-p}$. Conversely, assume that $u_{1}, u_{2} \in \mathcal{A}^{1}$, and $1<p<$ $\infty$, then for all $n \in \mathbb{I}$, we have

$$
\frac{1}{n} \sum_{k=1}^{n} u_{1}(k) \leq A_{1} u_{1}(k), \quad \text { and } \quad \frac{1}{n} \sum_{k=1}^{n} u_{2}(k) \leq A_{2} u_{2}(k) \text {, }
$$

holds for all $1 \leq k \leq n$. Thus, by letting $u=u_{1} u_{2}^{1-p}$, we have for all $n \in \mathbb{I}$ that

$$
\begin{aligned}
\frac{1}{n} \sum_{k=1}^{n} u(k) & \left(\frac{1}{n} \sum_{k=1}^{n} u^{\frac{1}{1-p}}(k)\right)^{p-1} \\
= & \left(\frac{1}{n} \sum_{k=1}^{n} u_{1}(k) u_{2}^{1-p}(k)\right)\left(\frac{1}{n} \sum_{k=1}^{n} u_{1}^{\frac{1}{1-p}}(k) u_{2}(k)\right)^{p-1} \\
\leq & {\left[\frac{1}{n} \sum_{k=1}^{n} u_{1}(k)\left(\frac{1}{A_{2}} \frac{1}{n} \sum_{k=1}^{n} u_{2}(k)\right)^{1-p}\right] } \\
& \times\left[\frac{1}{n} \sum_{k=1}^{n}\left(\frac{1}{A_{1}} \frac{1}{n} \sum_{k=1}^{n} u_{1}(k)\right)^{\frac{1}{1-p}} u_{2}(k)\right]^{p-1} \\
= & A_{1} A_{2}^{p-1}\left[\left(\frac{1}{n} \sum_{k=1}^{n} u_{1}(k)\right)\left(\frac{1}{n} \sum_{k=1}^{n} u_{2}(k)\right)^{1-p}\right] \\
& \times\left[\left(\frac{1}{n} \sum_{k=1}^{n} u_{1}(k)\right)^{-1}\left(\frac{1}{n} \sum_{k=1}^{n} u_{2}(k)\right)^{p-1}\right] \\
= & A_{1} A_{2}^{p-1},
\end{aligned}
$$

that is $u=u_{1} u_{2}^{1-p} \in \mathcal{A}^{p}$, with $[u]_{\mathcal{A}^{p}}=\left[u_{1}\right]_{\mathcal{A}^{1}}\left[u_{2}\right]_{\mathcal{A}^{1}}^{p-1}$. The proof is complete.

Theorem 9 Let $u$ be a positive weight and $p$ be a nonnegative real number. Then following statement holds: $u \in \mathcal{A}^{p}$ if and only if $u$ and $u^{\frac{1}{1-p}}$ are in $\mathcal{A}^{\infty}$. 
Proof We prove this property by using by Property (3) in Theorem 5 . Since

$$
\mathcal{A}^{\infty}=\bigcup_{1 \leq p<\infty} \mathcal{A}^{p}
$$

it is clear that $u \in \mathcal{A}^{p}$, for any $p>1$, if and only if $u \in \mathcal{A}^{\infty}$. Now, we have by Theorem 4 that $u \in \mathcal{A}^{p}$ if and only if $u^{1-p^{\prime}}=u^{\frac{1}{1-p}} \in \mathcal{A}^{p^{\prime}}$. That is, by Property (1) in Theorem $5\left(\mathcal{A}^{p^{\prime}} \subset \mathcal{A}^{\infty}\right)$, $u \in \mathcal{A}^{p}$ if and only if $u^{\frac{1}{1-p}} \in \mathcal{A}^{\infty}$. The proof is complete.

The next theorem is a self-improving property of weights in the Muckenhoupt class.

Theorem 10 Let $u$ be a positive weight, $p$ be a nonnegative number. If $u \in \mathcal{A}^{p}, p>1$, then $u \in \mathcal{A}^{p-\epsilon}$, for some $\epsilon>0$.

Proof Let $u \in \mathcal{A}^{p}$, for $p>1$, then, for $A>1$ and all $n \in \mathbb{I}$, we have

$$
\frac{1}{n} \sum_{k=1}^{n} u(k) \leq A\left(\frac{1}{n} \sum_{k=1}^{n} u^{-p^{\prime} / p}(k)\right)^{-p / p^{\prime}} .
$$

By Theorem $4, u^{-p^{\prime} / p}=u^{1-p^{\prime}} \in \mathcal{A}^{p^{\prime}}$. Also, by Theorem $2, u^{-p^{\prime} / p} \in \mathcal{G}^{q}$ for some $q$, or equivalently

$$
\left(\frac{1}{n} \sum_{k=1}^{n} u^{-p^{\prime} q / p}(k)\right)^{1 / q} \leq G \frac{1}{n} \sum_{k=1}^{n} u^{-p^{\prime} / p}(k) .
$$

By using (22) and (23), we have

$$
\left(\frac{1}{n} \sum_{k=1}^{n} u^{-p^{\prime} q / p}(k)\right)^{1 / q} \leq G\left(A^{-1} \frac{1}{n} \sum_{k=1}^{n} u(k)\right)^{-p^{\prime} / p},
$$

and then

$$
\frac{1}{n} \sum_{k=1}^{n} u(k) \leq G^{p / p^{\prime}} A\left(\frac{1}{n} \sum_{k=1}^{n} u^{-p^{\prime} q / p}(k)\right)^{-p / p^{\prime} q} .
$$

This follows on taking $p-\epsilon-1=p /\left(p^{\prime} q\right)$, or equivalently, taking $\epsilon=\frac{p-1}{q^{\prime}}$. Then $u \in \mathcal{A}^{p-\epsilon}$ for $p>1$ and some $\epsilon>0$. The proof is complete.

\section{Some basic properties of Gehring weights}

In this section, we prove some basic properties of Gehring weights. In the next lemma, we present the inclusion relation of Gehring classes $\mathcal{G}^{q}$ in $\mathcal{G}^{1}$-class of weights for all $1<q<\infty$.

Lemma 11 Let $u$ be a positive weight and $q$ be a nonnegative number. If $u \in \mathcal{G}^{q}$ for $q>1$, then

$$
\exp \left(\frac{1}{n} \sum_{k=1}^{n} \frac{u(k)}{\frac{1}{n} \sum_{k=1}^{n} u(k)} \log \left(\frac{u(k)}{\frac{1}{n} \sum_{k=1}^{n} u(k)}\right)\right)<\infty,
$$

holds. 
Proof Assume that $u \in \mathcal{G}^{q}$, then there exists $G>1$ such that

$$
\left[\left(\frac{1}{n} \sum_{k=1}^{n} u^{q}(k)\right)^{1 / q}\left(\frac{1}{n} \sum_{k=1}^{n} u(k)\right)^{-1}\right]^{q /(q-1)} \leq G,
$$

holds for all $n \in \mathbb{I}$, or equivalently

$$
\left(\frac{1}{n} \sum_{k=1}^{n}\left(\frac{u(k)}{\frac{1}{n} \sum_{k=1}^{n} u(k)}\right)^{q}\right)^{1 /(q-1)} \leq G,
$$

holds for all $n \in \mathbb{I}$. By taking the limit as $q$ tends to 1 and applying L'Hôpital's rule, we obtain

$$
\begin{aligned}
G & \geq \lim _{q \rightarrow 1}\left(\frac{1}{n} \sum_{k=1}^{n}\left(\frac{u(k)}{\frac{1}{n} \sum_{k=1}^{n} u(k)}\right)^{q}\right)^{1 /(q-1)} \\
& =\exp \left(\frac{1}{n} \sum_{k=1}^{n} \frac{u(k)}{\frac{1}{n} \sum_{k=1}^{n} u(k)} \log \left(\frac{u(k)}{\frac{1}{n} \sum_{k=1}^{n} u(k)}\right)\right) .
\end{aligned}
$$

The proof is complete.

In the next theorem, we present some basic inclusion properties of weights in the Gehring class.

Theorem 12 Let $u$ be a positive weight and $p$ and $q$ be real nonnegative numbers such that $p, q>1$. The following properties hold:

(1) $\mathcal{G}^{\infty} \subset \mathcal{G}^{q} \subset \mathcal{G}^{1}$ for all $1<q \leq \infty$.

(2) $\mathcal{G}^{q} \subset \mathcal{G}^{p}$ for all $1<p \leq q$.

(3) $\mathcal{G}^{1}=\bigcup_{1<q \leq \infty} \mathcal{G}^{q}$ with $[u]_{\mathcal{G}^{1}}=\lim _{q \rightarrow 1}[u]_{\mathcal{G}^{q}}$.

Proof (1) Assume that $u \in \mathcal{G}^{\infty}$, then, by the definition of $\mathcal{G}^{\infty}$, there exists $0<C<\infty$ such that, for all $n \in \mathbb{I}$, we have

$$
\sup _{1 \leq k \leq n} u(k) \leq C\left(\frac{1}{n} \sum_{k=1}^{n} u(k)\right),
$$

or equivalently

$$
u(k) \leq C\left(\frac{1}{n} \sum_{k=1}^{n} u(k)\right),
$$

for all $1 \leq k \leq n$. By applying (24) for all $1<q<\infty$, we have

$$
\begin{aligned}
& \left(\frac{1}{n} \sum_{k=1}^{n} u^{q}(k)\right)^{1 / q}\left(\frac{1}{n} \sum_{k=1}^{n} u(k)\right)^{-1} \\
& \leq\left[\frac{1}{n} \sum_{k=1}^{n}\left(C\left(\frac{1}{n} \sum_{k=1}^{n} u(k)\right)\right)^{q}\right]^{1 / q}\left(\frac{1}{n} \sum_{k=1}^{n} u(k)\right)^{-1}
\end{aligned}
$$




$$
=C\left(\frac{1}{n} \sum_{k=1}^{n} u(k)\right)\left(\frac{1}{n} \sum_{k=1}^{n} u(k)\right)^{-1}=C .
$$

That is, $u \in \mathcal{G}^{q}$ and hence $\mathcal{G}^{\infty} \subset \mathcal{G}^{q}$. Now, the inclusion $\mathcal{G}^{q} \subset \mathcal{G}^{1}$ is proved in Lemma 11 . This is the desired result.

(2) Assume $u \in \mathcal{G}^{q}$. Then there exists $G>1$ such that, for all $n \in \mathbb{I}$,

$$
\left(\frac{1}{n} \sum_{k=1}^{n} u^{q}(k)\right)^{1 / q} \leq G \frac{1}{n} \sum_{k=1}^{n} u(k) .
$$

Lemma 1 implies that $\mathcal{M}_{p} u \leq \mathcal{M}_{q} u$ for all $p \leq q$. Then, by substituting in (25), we have

$$
\left(\frac{1}{n} \sum_{k=1}^{n} u^{p}(k)\right)^{1 / p} \leq\left(\frac{1}{n} \sum_{k=1}^{n} u^{q}(k)\right)^{1 / q} \leq G \frac{1}{n} \sum_{k=1}^{n} u(k) .
$$

That is, $u \in \mathcal{G}^{p}$, which completes the proof of the second case.

(3) Property (1) states that, for all $1<q \leq \infty$, we have $\mathcal{G}^{q} \subset \mathcal{G}^{1}$, and then

$$
\bigcup_{1<q \leq \infty} \mathcal{G}^{q} \subseteq \mathcal{G}^{1}
$$

Conversely, assume that $u \in \mathcal{G}^{1}$ and assume, on the contrary, for all $1<q \leq \infty$, that $u \notin \mathcal{G}^{q}$. That is, for all $n \in \mathbb{I}$,

$$
\sup _{n \in \mathbb{I}}\left[\left(\frac{1}{n} \sum_{k=1}^{n} u^{q}(k)\right)^{1 / q}\left(\frac{1}{n} \sum_{k=1}^{n} u(k)\right)^{-1}\right]^{q /(q-1)}=\infty .
$$

By taking the limit of both sides of (27) as $q$ tends to 1 , we have

$$
\sup _{n \in \mathbb{I}}\left[\exp \left(\frac{1}{n} \sum_{k=1}^{n}\left(\frac{u(k)}{\frac{1}{n} \sum_{k=1}^{n} u(k)}\right) \log \left(\frac{u(k)}{\frac{1}{n} \sum_{k=1}^{n} u(k)}\right)\right)\right]=\infty \text {. }
$$

This contradicts the assumption $u \in \mathcal{G}^{1}$, which implies that, for some $1<q \leq \infty, u \in \mathcal{G}^{q}$, and

$$
\mathcal{G}^{1} \subseteq \bigcup_{1<q \leq \infty} \mathcal{G}^{q}
$$

By (26) and (28), we have $\mathcal{G}^{1}=\bigcup_{1<q \leq \infty} \mathcal{G}^{q}$. Furthermore, by applying L'Hôpital's rule and limit rules, we have

$$
\begin{aligned}
\lim _{q \rightarrow 1}[u]_{\mathcal{G}^{q}} & =\lim _{q \rightarrow 1} \sup _{n \in \mathbb{I}}\left[\left(\frac{1}{n} \sum_{k=1}^{n} u^{q}(k)\right)^{1 / q}\left(\frac{1}{n} \sum_{k=1}^{n} u(k)\right)^{-1}\right]^{q /(q-1)} \\
& =\sup _{n \in \mathbb{I}} \lim _{q \rightarrow 1}\left[\left(\frac{1}{n} \sum_{k=1}^{n} u^{q}(k)\right)^{1 / q}\left(\frac{1}{n} \sum_{k=1}^{n} u(k)\right)^{-1}\right]^{q /(q-1)}
\end{aligned}
$$




$$
\begin{aligned}
& =\sup _{n \in \mathbb{I}}\left[\exp \left(\frac{1}{n} \sum_{k=1}^{n}\left(\frac{u(k)}{\frac{1}{n} \sum_{k=1}^{n} u(k)}\right) \log \left(\frac{u(k)}{\frac{1}{n} \sum_{k=1}^{n} u(k)}\right)\right)\right] \\
& =[u]_{\mathcal{G}^{1}},
\end{aligned}
$$

which is the desired result of the case (3). The proof is complete.

Theorem 13 Let $u$ be a positive weight, $p$ be a nonnegative number. If $u \in \mathcal{A}^{p}$, then $u^{-1} \in$ $\mathcal{G}^{p^{\prime}-1}$.

Proof Assume that $u \in \mathcal{A}^{p}$, then

$$
\frac{1}{n} \sum_{k=1}^{n} u(k) \leq A\left(\frac{1}{n} \sum_{k=1}^{n} u^{1 /(1-p)}(k)\right)^{1-p}
$$

By Lemma 1 , we have $\mathcal{M}_{-1} u \leq \mathcal{M}_{1} u$, then (29) becomes

$$
\left(\frac{1}{n} \sum_{k=1}^{n}\left(u^{-1}(k)\right)^{1 /(p-1)}\right)^{p-1} \leq A \frac{1}{n} \sum_{k=1}^{n} u^{-1}(k) .
$$

That is, $u^{-1} \in \mathcal{G}^{p^{\prime}-1}$. This completes our proof.

In the next theorem, we prove the self-improving property of Gehring classes.

Theorem 14 Let $u$ be a positive weight, $q$ be a nonnegative number. If $u \in \mathcal{G}^{q}$ for $q>1$, then $u \in \mathcal{G}^{q+\epsilon}$, for some $\epsilon>0$.

Proof Assume that $u \in \mathcal{G}^{q}$ for $q>1$, then

$$
\left(\frac{1}{n} \sum_{k=1}^{n} u^{q}(k)\right)^{1 / q} \leq G \frac{1}{n} \sum_{k=1}^{n} u(k)
$$

By applying Theorem 2 we get $u \in \mathcal{A}^{p}$ for some $p$, and property (2) in Theorem 6 implies that $u^{\alpha} \in \mathcal{A}^{p}$ for $\alpha>1$ the smallest number satisfying $u \in \mathcal{G}^{\alpha}$ and $u^{1 /(1-p)} \in \mathcal{G}^{\alpha}$ (clearly, $q>\alpha$ ). Again, by applying Theorem 2 , then $u^{\alpha} \in \mathcal{G}^{s}$ for some $s$. Without loss of generality, we choose the largest $s$ satisfying $u^{\alpha} \in \mathcal{G}^{s}(A)$. Then, by using the condition $u \in \mathcal{G}^{\alpha}(B)$, it satisfies

$$
\left(\frac{1}{n} \sum_{k=1}^{n} u^{\alpha s}(k)\right)^{1 / \alpha s} \leq A^{1 / \alpha}\left(\frac{1}{n} \sum_{k=1}^{n} u^{\alpha}(k)\right)^{1 / \alpha} \leq A^{1 / \alpha} B \frac{1}{n} \sum_{k=1}^{n} u(k) .
$$

That is, $u \in \mathcal{G}^{\alpha s}$. The cases $q<s$ and $(q>s$ with $q<\alpha s$ ) are the only valid cases of $s$ and $q$ as otherwise there exists $q / \alpha>s$ satisfying the condition $u^{\alpha} \in \mathcal{G}^{q / \alpha}$, which contradicts the assumption. Then $u \in \mathcal{G}^{q+\epsilon}$ for some $\epsilon=\alpha s-q>0$. The proof is complete.

The next theorem presents the relation between the two classes $\mathcal{G}^{1}$ and $\mathcal{A}^{\infty}$. 
Theorem 15 The statement

$$
\mathcal{G}^{1}=\mathcal{A}^{\infty}=\bigcup_{1<p \leq \infty} \mathcal{A}^{p}=\bigcup_{1<q \leq \infty} \mathcal{G}^{q}
$$

holds.

Proof Assume that $u \in \mathcal{G}^{1}$, then, by Property (3) in Theorem 12, $u \in \mathcal{G}^{q}$ for some $1<$ $q \leq \infty$. By Theorem 2, this is true if and only if $u \in \mathcal{A}^{p}$ for some $p$, which implies that $u \in \mathcal{A}^{\infty}$. Hence, it is clear that $\mathcal{A}^{\infty}=\mathcal{G}^{1}$. The proof is complete.

\section{Some fundamental relations}

In this section, we prove some transition and inclusion relations involving the Muckenhoupt $\mathcal{A}^{\infty}$ and Gehring $\mathcal{G}^{q}$ classes.

Theorem 16 Let $u$ be a positive weight and $p_{0}$ be a real number. Then $u \in \mathcal{G}^{p_{0}}$ if and only if $u^{p_{0}} \in \mathcal{A}^{\infty}$.

Proof We start by assuming that $u^{p_{0}} \in \mathcal{A}^{\infty}=\bigcup_{p<\infty} \mathcal{A}^{p}$ for a fixed $1<p_{0}<\infty$, then $u^{p_{0}} \in$ $\mathcal{A}^{p}$, for some $1<p<\infty$. That is, for all $n \in \mathbb{I}$, there exists $1<A<\infty$, such that

$$
\frac{1}{n} \sum_{k=1}^{n} u^{p_{0}} \leq A\left(\frac{1}{n} \sum_{k=1}^{n} u^{\frac{p_{0}}{1-p}}\right)^{1-p} .
$$

Since $p_{0} /(1-p)<0<1$, Lemma 1 implies that

$$
\left(\frac{1}{n} \sum_{k=1}^{n} u^{\frac{p_{0}}{1-p}}\right)^{\frac{1-p}{p_{0}}} \leq \frac{1}{n} \sum_{k=1}^{n} u
$$

By substituting (31) into (30), we have

$$
\frac{1}{n} \sum_{k=1}^{n} u^{p_{0}} \leq A\left(\frac{1}{n} \sum_{k=1}^{n} u\right)^{p_{0}},
$$

or equivalently

$$
\left(\frac{1}{n} \sum_{k=1}^{n} u^{p_{0}}\right)^{1 / p_{0}} \leq A^{1 / p_{0}}\left(\frac{1}{n} \sum_{k=1}^{n} u\right) .
$$

Hence, $u \in \mathcal{G}^{p_{0}}$. Conversely, if we assume that $u \in \mathcal{G}^{p_{0}}$, for a fixed $1<p_{0}<\infty$. Then there exists $1<G<\infty$, such that for all $n \in \mathbb{I}$

$$
\left(\frac{1}{n} \sum_{k=1}^{n} u^{p_{0}}\right)^{1 / p_{0}} \leq G\left(\frac{1}{n} \sum_{k=1}^{n} u\right) .
$$


Theorem 14 implies that $u \in \mathcal{A}^{\infty}$. That is, there exists $1<A<\infty$, such that, for all $n \in \mathbb{I}, u$ satisfies

$$
\left(\frac{1}{n} \sum_{k=1}^{n} u\right)\left(\exp \frac{1}{n} \sum_{k=1}^{n} \log \frac{1}{u}\right) \leq A
$$

Then, by using (32) and (33), since $p_{0}>1$, we have

$$
\begin{aligned}
& \left(\frac{1}{n} \sum_{k=1}^{n} u^{p_{0}}\right)\left(\exp \frac{1}{n} \sum_{k=1}^{n} \log \frac{1}{u^{p_{0}}}\right) \\
& \quad=\left(\frac{1}{n} \sum_{k=1}^{n} u^{p_{0}}\right)\left(\exp \frac{1}{n} \sum_{k=1}^{n} \log \frac{1}{u}\right)^{p_{0}} \\
& \leq G^{p_{0}}\left(\frac{1}{n} \sum_{k=1}^{n} u\right)^{p_{0}}\left(\exp \frac{1}{n} \sum_{k=1}^{n} \log \frac{1}{u}\right)^{p_{0}} \leq(G A)^{p_{0}} .
\end{aligned}
$$

That is, $u^{p_{0}} \in \mathcal{A}^{\infty}$, which completes our proof.

Now, we give a quantitative version of the above theorem, which gives some relations between the norms of the classes $\mathcal{A}^{\infty}$ and $\mathcal{G}^{p}$.

Theorem 17 Let $u$ be a positive weight and $1<p<\infty$. Then

$$
\frac{\left[u^{p}\right]_{\mathcal{A}^{\infty}}^{1 / p}}{[u]_{\mathcal{A}^{\infty}}} \leq[u]_{\mathcal{G}^{p}}^{1 / p^{\prime}} \leq\left[u^{p}\right]_{\mathcal{A}^{\infty}}^{1 / p} .
$$

Proof By the definition of $[u]_{\mathcal{G}^{p}}$, we have for all $n \in \mathbb{I}$

$$
\frac{1}{n} \sum_{k=1}^{n} u^{p} \leq[u]_{\mathcal{G}^{p}}^{p / p^{\prime}}\left[\frac{1}{n} \sum_{k=1}^{n} u\right]^{p}
$$

By multiplying both sides by $\left((1 / n) \sum_{k=1}^{n} u^{p\left(1-q^{\prime}\right)}\right)^{q-1}$, for $q<\infty$, we obtain

$$
\frac{1}{n} \sum_{k=1}^{n} u^{p}\left(\frac{1}{n} \sum_{k=1}^{n} u^{p\left(1-q^{\prime}\right)}\right)^{q-1} \leq[u]_{\mathcal{G}^{p}}^{p / p^{\prime}}\left[\frac{1}{n} \sum_{k=1}^{n} u\left(\frac{1}{n} \sum_{k=1}^{n} u^{p\left(1-q^{\prime}\right)}\right)^{\frac{q-1}{p}}\right]^{p} .
$$

By taking the supremum of (35) over all $n \in \mathbb{I}$, we have

$$
\sup _{n \in \mathbb{I}} \frac{1}{n} \sum_{k=1}^{n} u^{p}\left(\frac{1}{n} \sum_{k=1}^{n} u^{p\left(1-q^{\prime}\right)}\right)^{q-1} \leq[u]_{\mathcal{G}^{p}}^{p / p^{\prime}} \sup _{n \in \mathbb{I}}\left[\frac{1}{n} \sum_{k=1}^{n} u\left(\frac{1}{n} \sum_{k=1}^{n} u^{p\left(1-q^{\prime}\right)}\right)^{\frac{q-1}{p}}\right]^{p},
$$

that is,

$$
\left[u^{p}\right]_{\mathcal{A}^{q}} \leq[u]_{\mathcal{G}^{p}}^{p / p^{\prime}}\left(\mathcal{A}_{\frac{p+q-1}{p}}(u)\right)^{p} .
$$


By taking the limit as $q$ tends to $\infty$, we have

$$
\frac{\left[u^{p}\right]_{\mathcal{A}}^{1 / p}}{[u]_{\mathcal{A}^{\infty}}} \leq[u]_{\mathcal{G}^{p}}^{1 / p^{\prime}}
$$

which is the first inequality. For the second inequality, by the definition of $\left[u^{p}\right]_{\mathcal{A}^{q}}^{1 / p}$, we have for all $n \in \mathbb{I}$

$$
\frac{\left(\frac{1}{n} \sum_{k=1}^{n} u^{p}\right)^{1 / p}}{\frac{1}{n} \sum_{k=1}^{n} u}=\frac{\left(\frac{1}{n} \sum_{k=1}^{n} u^{p}\right)^{1 / p}\left(\frac{1}{n} \sum_{k=1}^{n} u^{\frac{p}{1-q}}\right)^{\frac{q-1}{p}}}{\frac{1}{n} \sum_{k=1}^{n} u\left(\frac{1}{n} \sum_{k=1}^{n} u^{\frac{p}{1-q}}\right)^{\frac{q-1}{p}}} .
$$

Now, by Jensen's inequality since $p /(1-q)<0<1$, we see that

$$
\frac{1}{n} \sum_{k=1}^{n} u^{\frac{p}{1-q}} \geq\left(\frac{1}{n} \sum_{k=1}^{n} u\right)^{\frac{p}{1-q}}
$$

which implies that

$$
\frac{1}{\frac{1}{n} \sum_{k=1}^{n} u\left(\frac{1}{n} \sum_{k=1}^{n} u^{\frac{p}{1-q}}\right)^{\frac{q-1}{p}}} \leq \frac{1}{\frac{1}{n} \sum_{k=1}^{n} u\left(\left(\frac{1}{n} \sum_{k=1}^{n} u\right)^{\frac{p}{1-q}}\right)^{\frac{q-1}{p}}} \leq 1 .
$$

By using this in (38), we get

$$
\frac{\left(\frac{1}{n} \sum_{k=1}^{n} u^{p}\right)^{1 / p}}{\frac{1}{n} \sum_{k=1}^{n} u} \leq\left(\frac{1}{n} \sum_{k=1}^{n} u^{p}\left(\frac{1}{n} \sum_{k=1}^{n} u^{\frac{p}{1-q}}\right)^{q-1}\right)^{1 / p} .
$$

Taking the supremum of (38) over all $n \in \mathbb{I}$, we have $[u]_{\mathcal{G}^{p}}^{1 / p^{\prime}} \leq\left[u^{p}\right]_{\mathcal{A}^{q}}^{1 / p}$. By taking the limit as $q$ tends to $\infty$, we have

$$
[u]_{\mathcal{G}^{p}}^{1 / p^{\prime}} \leq\left[u^{p}\right]_{\mathcal{A}^{\infty}}^{1 / p}
$$

The proof is complete.

Theorem 18 Let $u$ be a positive weight and $1<p<\infty$. Then

$$
\max \left\{[u]_{\mathcal{A}^{\infty}},\left[u^{1-p^{\prime}}\right]_{\mathcal{A}^{\infty}}^{p-1}\right\} \leq[u]_{\mathcal{A}^{p}} \leq[u]_{\mathcal{A}^{\infty}}\left[u^{1-p^{\prime}}\right]_{\mathcal{A}^{\infty}}^{p-1}
$$

Proof For $p \leq q$, we have $[u]_{\mathcal{A}^{p}} \geq[u]_{\mathcal{A}^{q}}$, and thus

$$
[u]_{\mathcal{A}^{\infty}} \leq[u]_{\mathcal{A}^{p}} .
$$


Furthermore, for $q<\infty$, we have

$$
\begin{aligned}
{\left[u^{1-p^{\prime}}\right]_{\mathcal{A}}^{p-1} } & =\sup _{n \in \mathbb{I}}\left\{\frac{1}{n} \sum_{k=1}^{n} u^{1-p^{\prime}}\left(\frac{1}{n} \sum_{k=1}^{n} u^{\left(1-p^{\prime}\right)\left(1-q^{\prime}\right)}\right)^{q-1}\right\}^{p-1} \\
& =\sup _{n \in \mathbb{I}}\left\{\frac{\frac{1}{n} \sum_{k=1}^{n} u\left(\frac{1}{n} \sum_{k=1}^{n} u^{1-p^{\prime}}\right)^{p-1}}{\frac{1}{n} \sum_{k=1}^{n} u\left(\frac{1}{n} \sum_{k=1}^{n} u^{\left(1-p^{\prime}\right)\left(1-q^{\prime}\right)}\right)^{-(1-p)(1-q)}}\right\} \\
& \leq \sup _{n \in \mathbb{I}} \frac{1}{n} \sum_{k=1}^{n} u\left(\frac{1}{n} \sum_{k=1}^{n} u^{1-p^{\prime}}\right)^{p-1}=[u]_{\mathcal{A}^{p}} .
\end{aligned}
$$

By taking the limit of (41) as $q$ tends to $\infty$, we have

$$
\left[u^{1-p^{\prime}}\right]_{\mathcal{A}^{\infty}}^{p-1} \leq[u]_{\mathcal{A}^{p}}
$$

By (40) and (42), then

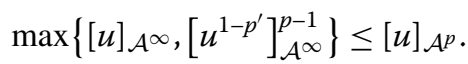

Now, for the second inequality, we have

$$
\begin{aligned}
\frac{1}{n} \sum_{k=1}^{n} u\left(\frac{1}{n} \sum_{k=1}^{n} u^{1-p^{\prime}}\right)^{p-1}= & \frac{1}{n} \sum_{k=1}^{n} u\left(\frac{1}{n} \sum_{k=1}^{n} u^{1-q^{\prime}}\right)^{q-1} \\
& \times\left(\frac{1}{n} \sum_{k=1}^{n} u^{1-p^{\prime}}\left(\frac{1}{n} \sum_{k=1}^{n} u^{1-q^{\prime}}\right)^{\frac{1-q}{p-1}}\right)^{p-1} .
\end{aligned}
$$

Since $1-q$ and $1-q^{\prime}<0$, and since (see Lemma 1 )

$$
\left(\frac{1}{n} \sum_{k=1}^{n} u^{-1}\right)^{-1} \leq \frac{1}{n} \sum_{k=1}^{n} u
$$

we have

$$
\begin{aligned}
\frac{1}{n} \sum_{k=1}^{n} u^{1-p^{\prime}}\left(\frac{1}{n} \sum_{k=1}^{n} u^{1-q^{\prime}}\right)^{\frac{1-q}{p-1}} & \leq \frac{1}{n} \sum_{k=1}^{n} u^{1-p^{\prime}}\left(\frac{1}{n} \sum_{k=1}^{n} u^{q^{\prime}-1}\right)^{\frac{q-1}{p-1}} \\
& =\frac{1}{n} \sum_{k=1}^{n} u^{1-p^{\prime}}\left(\frac{1}{n} \sum_{k=1}^{n} u^{\left(1-p^{\prime}\right)\left(q^{\prime}-1\right) /\left(1-p^{\prime}\right)}\right)^{\frac{q-1}{p-1}}
\end{aligned}
$$

By setting $r-1=(q-1) /(p-1)$, we have

$$
r^{\prime}-1=\frac{1}{r-1}=\frac{p-1}{q-1}=\frac{q^{\prime}-1}{p^{\prime}-1} .
$$


Hence, from (43) and (44), we have

$$
\begin{aligned}
\frac{1}{n} \sum_{k=1}^{n} u\left(\frac{1}{n} \sum_{k=1}^{n} u^{1-p^{\prime}}\right)^{p-1} \leq & \frac{1}{n} \sum_{k=1}^{n} u\left(\frac{1}{n} \sum_{k=1}^{n} u^{1-q^{\prime}}\right)^{q-1} \\
& \times\left[\frac{1}{n} \sum_{k=1}^{n} u^{1-p^{\prime}}\left(\frac{1}{n} \sum_{k=1}^{n} u^{\left(1-p^{\prime}\right)\left(1-r^{\prime}\right)}\right)^{r-1}\right]^{p-1} .
\end{aligned}
$$

Thus by taking the supremum over all $n \in \mathbb{I}$, we have

$$
[u]_{\mathcal{A}^{p}} \leq[u]_{\mathcal{A}^{q}}\left[u^{1-p^{\prime}}\right]_{\mathcal{A}^{r}}^{p-1}
$$

By taking the limit of both sides of (45) as $q$ tends to $\infty$, we have

$$
[u]_{\mathcal{A}^{p}} \leq[u]_{\mathcal{A}^{\infty}}\left[u^{1-p^{\prime}}\right]_{\mathcal{A}^{\infty}}^{p}
$$

The proof is complete.

\section{Acknowledgements}

The authors thank the referees for valuable comments that improve the presentation of the results in this paper. The third author is highly thankful to Prince Sultan University for supporting this work through the research group Nonlinear Analysis Methods in Applied Mathematics (NAMAM) group number RG-DES-2017-01-17.

Funding

Not applicable.

Availability of data and materials

Not applicable.

\section{Ethics approval and consent to participate}

Not applicable.

\section{Competing interests}

The authors declare that they have no competing interests.

\section{Consent for publication}

Not applicable.

\section{Authors' contributions}

All authors have taken equal part in this research and they read and approved the final manuscript.

\section{Author details}

${ }^{1}$ Mathematics Division, Faculty of Advanced Basic Sciences, Galala University, Galala New City, Suez, Egypt. ${ }^{2}$ Department of Mathematics, Faculty of Science, Mansoura University, 35516 Mansoura, Egypt. ${ }^{3}$ Department of Mathematics and General Sciences, Prince Sultan University, 11586 Riyadh, Saudi Arabia. ${ }^{4}$ School of Mathematics, Statistics and Applied Mathematics, National University of Ireland, Galway, Ireland. ${ }^{5}$ Department of Mathematics, Texas A \& M University-Kingsville, 78363 Texas, USA.

\section{Publisher's Note}

Springer Nature remains neutral with regard to jurisdictional claims in published maps and institutional affiliations.

Received: 26 August 2020 Accepted: 5 November 2020 Published online: 02 January 2021

\section{References}

1. Basile, L., D'Apuzzo, L., Squillante, M.: The limit class of Gehring type $G_{\infty}$. Boll. Unione Mat. Ital. (9) 11, $871-884$ (1997)

2. Bojarski, B., Sbordone, C., Wik, I.: The Muckenhoupt class $A_{1}(\mathbb{R})$. Stud. Math. 101, 155-163 (1992)

3. Böttcher, A., Spitkovsky, I.: Wiener-Hopf integral operators with PC symbols on spaces with Muckenhoupt weight. Rev. Mat. Iberoam. 9, 257-279 (1993)

4. Böttcher, A., Seybold, M.: Wackelsatz and Stechkin's inequality for discrete Muckenhoupt weights. Tech. Univ. Chemnitz 99, 7 (1999) 
5. Böttcher, A., Seybold, M.: Discrete Wiener-Hopf operators on spaces with Muckenhoupt weight. Stud. Math. 143, $121-144(2000)$

6. Böttcher, A., Seybold, M.: Discrete one-dimensional zero-order pseudodifferential operators on spaces with Muckenhoupt weight. Algebra Anal. 13, 116-129 (2001)

7. Böttcher, A., Spitkovsky, I.: Pseudodifferential operators with heavy spectrum. Integral Equ. Oper. Theory 19, 251-269 (1994)

8. Coifman, R.R., Fefferman, C.: Weighted norm inequalities for maximal functions and singular integrals. Stud. Math. 51, 241-250 (1974)

9. Corporente, R.: Weighted Integral Inequalities, BMO-spaces and applications. Dissertation thesis (2007)

10. D'Apuzzo, L., Sbordone, C.: Reverse Hölder inequalities, a sharp result. Rend. Lincei Mat. Appl. 10, 357-366 (1990)

11. Dindoš, M., Wall, T.: The sharp $A^{p}$ constant for weights in a reverse Hölder class. Rev. Mat. Iberoam. 25, 559-594 (2009)

12. Garcia-Cuerva, J., Rubio de Francia, J.L.: Weighted Norm Inequalities and Related Topics. North-Holland Mathematics Studies, vol. 116. North-Holland, Amsterdam (1985)

13. Gehring, F.W.: The $L^{p}$-integrability of the partial derivatives of a quasiconformal mapping. Bull. Am. Math. Soc. 97, 465-466 (1973)

14. Gehring, F.W.: The $L^{p}$-integrability of the partial derivatives of a quasi-conformal mapping. Acta Math. 130, 265-277 (1973)

15. Hunt, R., Muckenhoupt, B., Wheeden, R.: Weighted norm inequalities for the conjugate function and Hilbert transform. Trans. Am. Math. Soc. 176, 227-251 (1973)

16. Johnson, R., Neugebauer, C.J.: Homeomorphisms preserving Ap. Rev. Mat. Iberoam. 3(2), 249-273 (1987)

17. Korenovskii, A.A.: The exact continuation of a reverse Hölder inequality and Muckenhoupt's conditions. Math. Notes 52, 1192-1201 (1992)

18. Korenovskii, A.A., Fomichev, V.V.: Self-improvement of summability factors of functions satisfying the reverse Hölder inequality in limit cases. Ukr. Math. J. 62, 552-563 (2010)

19. Magyar, A., Stein, E.M., Wainger, S.: Discrete analogues in harmonic analysis: spherical averages. Ann. Math. 155, 189-208 (2002)

20. Malaksiano, N.A.: The exact inclusion of Gehring classes in Muckenhoupt classes. Mat. Zametki 70, 742-750 (2001) (in Russian), English translation in Mathematical Notes 70, 673-681 (2001)

21. Malaksiano, N.A.: The precise embeddings of one-dimensional Muckenhoupt classes in Gehring classes. Acta Sci. Math. 68, 237-248 (2002)

22. Mucheknhoupt, B.: Weighted norm inequalities for the Hardy maximal function. Trans. Am. Math. Soc. 165, 207-226 (1972)

23. Popoli, A.: Sharp integrability exponents and constants for Muckenhoupt and Gehring weights as solutions to a unique equation. Ann. Acad. Sci. Fenn., Math. 43, 785-805 (2018)

24. Saker, S.H., Krnić, M., Pečarić, J.: Higher summability theorems from the weighted reverse discrete inequalities. Appl. Anal. Discrete Math. 13, 423-439 (2019)

25. Saker, S.H., Kubiaczyk, I.: Higher summability and discrete weighted Muckenhoupt and Gehring type inequalities. Proc. Edinb. Math. Soc. 62, 949-973 (2019)

26. Saker, S.H., Mahmoud, R.R.: Embeddings of discrete Muckenhoupt classes in discrete Gehring classes and the converse with applications. Submitted

27. Saker, S.H., O'Regan, D., Agarwal, R.P.: A higher integrability theorem from a reverse weighted inequality. Bull. Lond. Math. Soc. 51, 967-977 (2019)

28. Saker, S.H., Rabie, S.S., AlNemer, G., Zakarya, M.: On structure of discrete Muchenhoupt and discrete Gehring classes. J. Inequal. Appl. 2020, 233 (2020). https://doi.org/10.1186/s13660-020-02497-4

29. Vasyuinin, V:: The exact constant in the inverse Hölder inequality for Muckenhoupt weights. Algebra Anal. 15, 73-117 (2003) (in Russian), English translation in St. Petersburg Math. J. 15, 49-79 (2004)

\section{Submit your manuscript to a SpringerOpen ${ }^{\circ}$ journal and benefit from:}

- Convenient online submission

- Rigorous peer review

- Open access: articles freely available online

- High visibility within the field

- Retaining the copyright to your article

Submit your next manuscript at $\gg$ springeropen.com 